\title{
LUT
}

Lappeenranta

University of Technology

\section{Reduction of the width of particle size distribution to improve pressure filtration properties of slurries}

\author{
Kinnarinen Teemu, Tuunila Ritva, Häkkinen Antti
}

This is a Final draft version of a publication

published by Elsevier

in Minerals Engineering

DOI: $\quad 10.1016 /$ j.mineng.2016.12.009

Copyright of the original publication: (c) Elsevier 2017

Please cite the publication as follows:

Kinnarinen, T., Tuunila, R., Häkkinen, A., Reduction of the width of particle size distribution to improve pressure filtration properties of slurries, Minerals Engineering, 2017, 102: 68-74. DOI: 10.1016/j.mineng.2016.12.009 


\title{
Reduction of the width of particle size distribution to improve pressure filtration properties of slurries
}

\author{
Teemu Kinnarinen*', Ritva Tuunila, Antti Häkkinen \\ LUT School of Engineering Science \\ Lappeenranta University of Technology \\ P.O. Box 20, FI-53851 Lappeenranta, Finland \\ *Corresponding author, Tel. +358 405621398, E-mail teemu.kinnarinen@lut.fi
}

\begin{abstract}
The filtration properties of mineral slurries depend for instance on the properties of the solid particles and on the way how these particles arrange themselves when the filter cake is formed. In cake filtration applications, the focus is often on particle size instead of the shape of particle size distribution (PSD). The aim of this experimental study is to demonstrate the influence of the width of PSD on the properties of the filter cake in the pressure filtration of $\mathrm{Ni}-\mathrm{Cu}$ mine tailings. Modification of PSD is performed by using a stirred media mill at various operational conditions. The results show that not only particle size should be considered when the filtration properties are evaluated: a significant reduction of the average specific cake resistance (> $60 \%$ ) and increase of the average porosity (almost $30 \%$ ) can be achieved by stirred media grinding at optimal conditions, in spite of reducing the median particle size. On the other hand, the selection of unfavorable grinding conditions may cause substantial increase in cake resistance. Above all, the results imply that the size of particles in the coarse end of PSD should be reduced while avoiding grinding of the finest particles. This seems to be obtainable by paying special attention to the size of the grinding medium. The main outcomes of this study will help in reducing the energy consumption of dewatering of tailings to be disposed of, and recovering valuable elements from tailings deposited in ponds, requiring the use of optimized grinding circuits and solid-liquid separation operations. However, the benefits obtained in the filtration stage should overcome the increased costs caused by the installation and energy consumption of the grinding systems. This means that the demonstrated grinding method may be profitably utilized in industrial scale only when the material is easy to grind and the filtration properties can be significantly improved, or when the filtration plant is the main bottleneck of the process.
\end{abstract}

\section{Introduction}

Wet grinding operations in minerals processing produce slurries where particle size distribution (PSD) of solids is optimized with respect to the quality of the final product and requirements of the subsequent unit operations, such as concentration by froth flotation and dewatering. Solid-liquid separation, performed for instance with vacuum or pressure filters, is routinely carried out before thermal drying of the product. The filter cake is basically a porous bed consisting of particles of non-uniform sizes and shapes and pores between them. The pores, i.e. voids, of the bed form a complex, randomly arranged network which is difficult to characterize with respect to size and shape (Lin and Miller, 2000a,b, 2004). The importance of particle size and shape in cake filtration is widely recognized in the literature. Small particles are known to cause high resistance to filtration (Wakeman, 2007), which increases the cost of separation and drying (Basim and Khalili, 2015), 
and some non-spherical particle shapes have been reported to increase the compressibility and porosity of the filter cake (Bourcier et al., 2016). Among the most common statistical particle sizes used to describe the particle size of solids are the surface area -based Sauter mean diameter D[3,2], the median diameter $\mathrm{D}_{50}$, and the volumetric mean diameter $\mathrm{D}[4,3]$ (Allen, 2003). The basic equations relating the properties of the filter cake to its permeability, such as the Kozeny-Carman equation (see e.g. Svarovsky, 1981; Ripperger et al., 2012; Osterroth et al., 2016), include not only the (surface area -based) particle size of the solids but also average bed porosity as key parameters. The assumption of monosized spherical particles forming an incompressible cake is typically used. When using these equations for experimental data the most influential reasons behind changes in cake porosity, such as the width of PSD and the particle shape distribution, are often not discussed at all. Too little attention has been paid to the influence of the particle size distribution on the separation characteristics of industrial mineral suspensions. Additionally, the effects of grinding parameters on the filtration properties of the suspension have been rarely discussed in the literature.

The main objective of this paper is to investigate the effect of the reduction of particle size on pressure filtration properties. The study aims at demonstrating that the reduced width of particle size distribution can help to overcome the negative effect on filtration caused by fine particles, and lead to a significantly improved filtration performance. An industrial sample of mine tailings is used as model suspension in order to ensure the practical relevance of the study.

\section{Materials and methods}

\subsection{Characterization of slurry}

The slurry sample was received from a nickel-copper mine. Table 1 presents the main characteristics of the slurry, including the density of the slurry and solids, the solids content of the slurry, and selected particle sizes of volumetric undersize distribution. The density of the slurry was measured on the basis of the mass and volume of the slurry sample, the solids content of the slurry was measured by drying the slurry at $105{ }^{\circ} \mathrm{C}$ to dryness, and the density of the solids was determined by using a liquid pycnometer. All particle size distributions presented in this paper were measured with a Malvern Mastersizer 3000 laser diffraction particle size analyzer.

Table 1. Main characteristics of the tailings slurry.

\begin{tabular}{llllllll}
\hline $\begin{array}{l}\rho_{\text {slurry }} \\
\left(\mathrm{kg} / \mathrm{m}^{3}\right)\end{array}$ & $\begin{array}{l}C_{s} \\
(\mathrm{wt} . \%)\end{array}$ & $\begin{array}{l}\rho_{s} \\
\left(\mathrm{~kg} / \mathrm{m}^{3}\right)\end{array}$ & $\begin{array}{l}D_{10} \\
(\mu \mathrm{m})\end{array}$ & $\begin{array}{l}D[3,2] \\
(\mu \mathrm{m})\end{array}$ & $\begin{array}{l}D_{50} \\
(\mu \mathrm{m})\end{array}$ & $\begin{array}{l}D_{[4,3]} \\
(\mu \mathrm{m})\end{array}$ & $\begin{array}{l}D_{90} \\
(\mu \mathrm{m})\end{array}$ \\
\hline 1098 & 14.9 & 3130 & 1.73 & 4.71 & 13.9 & 24.0 & 61.6 \\
\hline
\end{tabular}

The variations in the size and shape of particles in a dried slurry sample are illustrated by an image (Fig. 1) taken with a JEOL JSM-5800 scanning electron microscope. As can be observed, the particle shapes are very irregular and differ considerably from the perfectly spherical shape that is assumed in most theoretical approaches related to cake filtration. The particle shape distributions of selected samples, measured with Malvern Morphologi G3, are discussed in Section 4.4. 


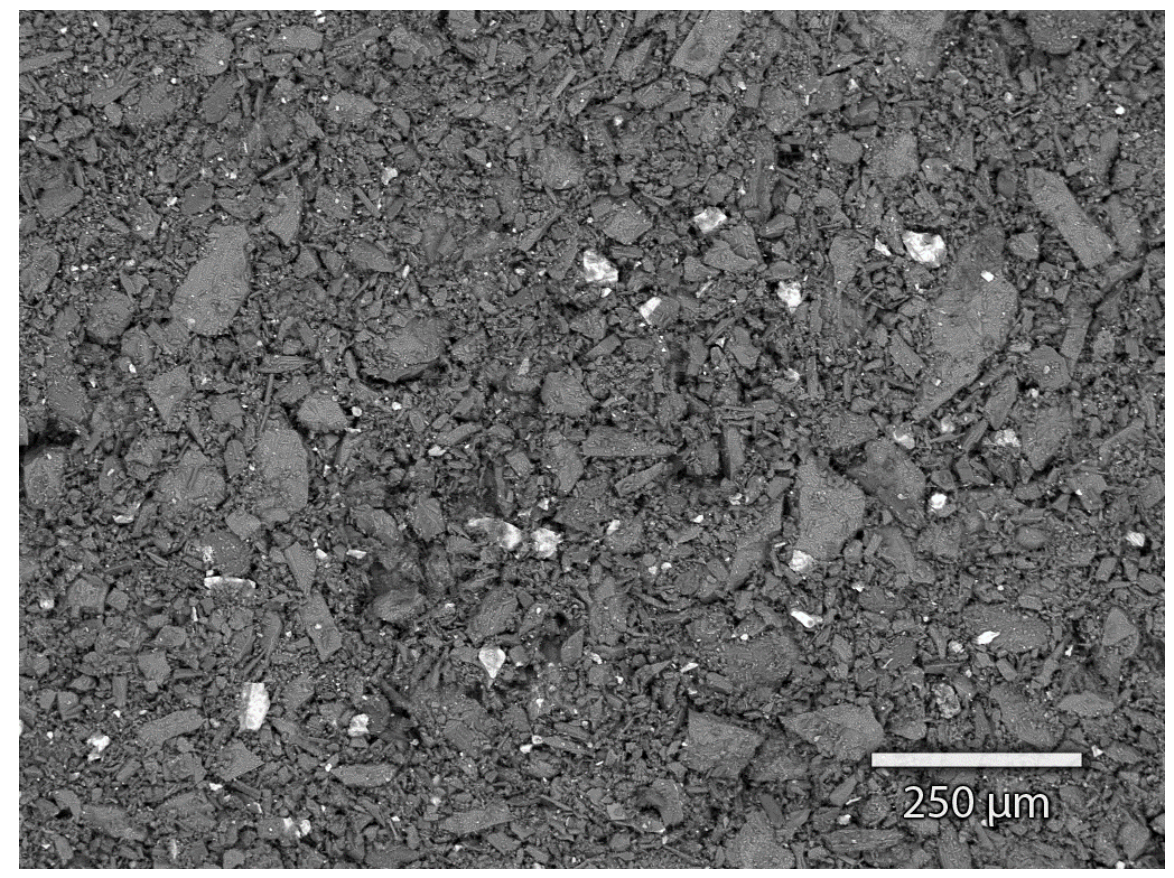

Fig. 1. SEM image of a dried slurry sample.

\section{2. $\quad$ Grinding with a stirred media mill}

The particle size distribution of the slurry solids was modified by using a vertical stirred media mill (Vollrath Salomix) described in closer detail by Kinnarinen et al. (2015). The experimental variables included 1) diameter of the glass beads (1-3 mm), 2) grinding time (5-15 min), and 3) stirring speed (300-700 rpm). The volume of the grinding vessel was approximately $5 \mathrm{dm}^{3}$, and the mass of slurry and glass beads in all tests was 2.2 and $3 \mathrm{~kg}$, respectively. The total number of experiments was 31 . The experimental plan, as well as the obtained grinding results are shown in Table 2. The definitions of the particle mean diameters and specific surface area are given below in Section 3. In this paper, the influence of each grinding variable on the particle size is discussed only from the point of view of the subsequent pressure filtration.

Table 2. Grinding variables and results. $D_{10}, D_{50}, D_{90}$, are particle diameters describing the volumetric undersize distribution. Diameters $D[3,2]$ and $D[4,3]$ are the area-based and volume-based mean particle sizes, respectively. $S_{w}$ is the weight-based specific surface area calculated on the basis of the Sauter mean diameter $D[3,2]$ assuming that each particle is spherical and has a smooth surface.

\begin{tabular}{llllllllll}
\hline $\begin{array}{l}\text { Test } \\
(\#)\end{array}$ & $\begin{array}{l}\text { Bead } \\
\text { diameter } \\
(\mathrm{mm})\end{array}$ & $\begin{array}{l}\text { Grinding } \\
\text { time } \\
(\mathrm{min})\end{array}$ & $\begin{array}{l}\text { Stirring } \\
\text { speed } \\
(\mathrm{rpm})\end{array}$ & $\begin{array}{l}D_{10} \\
(\mu \mathrm{m})\end{array}$ & $\begin{array}{l}D[3,2] \\
(\mu \mathrm{m})\end{array}$ & $\begin{array}{l}D_{50} \\
(\mu \mathrm{m})\end{array}$ & $\begin{array}{l}D[4,3] \\
(\mu \mathrm{m})\end{array}$ & $\begin{array}{l}D_{90} \\
(\mu \mathrm{m})\end{array}$ & $\begin{array}{l}S_{w} \\
\left(\mathrm{~m}^{2} / \mathrm{kg}\right)\end{array}$ \\
\hline 1 & 2 & 10 & 500 & 1.42 & 3.65 & 7.17 & 12.7 & 30.6 & 525 \\
2 & 3 & 15 & 300 & 1.50 & 3.88 & 8.06 & 14.1 & 33.0 & 494 \\
3 & 3 & 5 & 300 & 1.44 & 3.76 & 8.10 & 13.2 & 31.3 & 510 \\
4 & 3 & 15 & 700 & 1.36 & 3.09 & 4.72 & 7.88 & 16.1 & 620 \\
\hline
\end{tabular}




\begin{tabular}{llllllllll}
5 & 2 & 10 & 500 & 1.47 & 3.70 & 7.33 & 13.2 & 31.9 & 518 \\
6 & 1 & 15 & 300 & 1.32 & 3.43 & 6.91 & 13.5 & 33.7 & 559 \\
7 & 3 & 5 & 700 & 1.54 & 3.85 & 7.71 & 12.5 & 28.0 & 498 \\
8 & 2 & 10 & 500 & 1.46 & 3.73 & 7.62 & 13.7 & 34.0 & 514 \\
9 & 1 & 15 & 700 & 1.25 & 3.12 & 5.32 & 13.5 & 32.8 & 614 \\
10 & 1 & 5 & 300 & 1.50 & 3.91 & 8.72 & 15.4 & 38.0 & 490 \\
11 & 1 & 5 & 700 & 1.44 & 3.74 & 7.87 & 15.8 & 39.7 & 512 \\
12 & 3 & 10 & 500 & 1.57 & 3.97 & 8.14 & 13.8 & 32.1 & 483 \\
13 & 1 & 10 & 500 & 1.37 & 3.59 & 7.50 & 15.5 & 39.4 & 534 \\
14 & 2 & 5 & 500 & 1.60 & 4.21 & 10.30 & 19.5 & 50.3 & 455 \\
15 & 2 & 10 & 700 & 1.33 & 3.31 & 5.88 & 11.3 & 26.0 & 579 \\
16 & 2 & 10 & 500 & 1.46 & 3.74 & 7.55 & 13.8 & 33.8 & 512 \\
17 & 2 & 10 & 300 & 1.41 & 3.69 & 7.79 & 15.1 & 37.2 & 519 \\
18 & 2 & 15 & 500 & 1.41 & 3.60 & 6.89 & 14.1 & 34.9 & 532 \\
19 & 2 & 5 & 300 & 1.48 & 3.91 & 8.95 & 17.0 & 42.7 & 490 \\
20 & 3 & 10 & 300 & 1.55 & 4.03 & 9.06 & 15.5 & 37.5 & 476 \\
21 & 1 & 10 & 300 & 1.39 & 3.71 & 8.31 & 16.0 & 40.4 & 517 \\
22 & 1 & 5 & 500 & 1.57 & 4.20 & 10.6 & 21.7 & 57.3 & 456 \\
23 & 2 & 10 & 500 & 1.52 & 3.94 & 8.45 & 17.0 & 42.6 & 486 \\
24 & 2 & 5 & 700 & 1.54 & 3.99 & 8.79 & 16.3 & 41.0 & 480 \\
25 & 2 & 15 & 300 & 1.40 & 3.65 & 7.66 & 15.4 & 39.1 & 525 \\
26 & 3 & 15 & 500 & 1.42 & 3.48 & 6.31 & 10.4 & 22.7 & 551 \\
27 & 3 & 10 & 700 & 1.42 & 3.43 & 6.05 & 10.3 & 21.9 & 559 \\
28 & 2 & 15 & 700 & 1.24 & 2.95 & 4.63 & 9.46 & 20.1 & 650 \\
29 & 3 & 5 & 500 & 1.62 & 4.19 & 9.81 & 16.0 & 38.3 & 457 \\
30 & 1 & 10 & 700 & 1.30 & 3.40 & 6.68 & 15.0 & 39.0 & 564 \\
31 & 1 & 15 & 500 & 1.37 & 3.65 & 7.90 & 17.3 & 46.1 & 525 \\
\hline & & & & & & & & &
\end{tabular}

\subsection{Pressure filtration}

The slurry samples were filtered at room temperature with a Nutsche pressure filter at pressure differences of 2, 4, and 6 bar. The mass of each batch of slurry fed into the cylindrical filter chamber with a total volume of $350 \mathrm{~cm}^{3}$ and a diameter of $52 \mathrm{~mm}$ was $250 \pm 1 \mathrm{~g}$. Discs made of pure cellulose (T-1000, Pall Corporation) were used as filter media after careful wetting in reverse osmosis quality water. An automatic data recording system was used to record the mass of the filtrate and the filtration pressure once a second during the filtration. After each test, the filter cake was dried in an oven at $105^{\circ} \mathrm{C}$ to total dryness in order to calculate the solid content and further also the porosity of the cake.

\section{Theory}

The Sauter mean diameter $D[3,2](\mathrm{m})$ and the volume mean diameter $D[4,3](\mathrm{m})$ are defined as (Allen, 2003): 


$$
\begin{gathered}
D[3,2]=\frac{\sum_{i=1}^{n} D_{i}^{3} v_{i}}{\sum_{i=1}^{n} D_{i}^{2} v_{i}} \\
D[4,3]=\frac{\sum_{i=1}^{n} D_{i}^{4} v_{i}}{\sum_{i=1}^{n} D_{i}^{3} v_{i}}
\end{gathered}
$$

where $D$ is the particle diameter (m) and $v_{i}$ is the proportion of particles in the size fraction.

When the volume-based specific surface area $S_{v}\left(\mathrm{~m}^{2} \mathrm{~m}^{-3}\right)$ is calculated for spherical particles using the data obtained by a particle size analyzer, the only particle property required for the calculation is the Sauter mean diameter:

$$
S_{v}=\frac{6}{D[3,2]}
$$

The weight-based specific surface area $S_{w}\left(\mathrm{~m}^{2} \mathrm{~kg}^{-1}\right)$ is calculated using Eq. (4).

$$
S_{w}=\frac{S_{v}}{\rho_{s}}
$$

The average specific cake resistance for constant pressure filtration is calculated with equations based on Darcy's law (Darcy, 1856). The flow rate through a filter cake and a filter medium is obtained from Eq. (5):

$$
Q=\frac{\Delta p A}{\alpha \mu w+\mu R_{m}}
$$

where $Q$ is the volumetric flow rate $\left(\mathrm{m}^{3} \mathrm{~s}^{-1}\right), \Delta p$ is the pressure difference $(\mathrm{Pa}), A$ is the filtration area $\left(\mathrm{m}^{2}\right), \alpha$ is the specific cake resistance $\left(\mathrm{m} \mathrm{kg}^{-1}\right), \mu$ is the viscosity of the liquid $(\mathrm{Pa} \mathrm{s}), w$ is the weight of the cake per unit area $\left(\mathrm{kg} \mathrm{m}^{-2}\right)$, and $R_{m}$ is the resistance of the filter medium $\left(\mathrm{m}^{-1}\right)$.

When the volume of filtrate at each moment of time is known, it is most convenient to use the integrated equation (Eq. 6) from which the average specific cake resistance can be solved.

$$
\frac{t}{V}=\frac{\alpha_{a v} \mu c}{2 A^{2} \Delta p} V+\frac{\mu R_{m}}{A \Delta p}
$$


where $t$ is time (s), $V$ is the volume of filtrate $\left(\mathrm{m}^{3}\right), \alpha_{a v}$ is the average specific cake resistance ( $\mathrm{m}$ $\left.\mathrm{kg}^{-1}\right)$, and $c$ is the mass of dry solids in cake per volume of filtrate $\left(\mathrm{kg} \mathrm{m}^{-3}\right)$. The procedure of determining $\alpha_{a v}$ from experimental data is widely applied, and has been introduced in the literature for instance by Tien (2012), Svarovsky, (1981), Concha (2014), and Tarleton and Wakeman (2007).

In the case of incompressible cakes, the average specific cake resistance can be related to the Sauter mean diameter $D[3,2]$ of particles and the average porosity of the filter cake $\varepsilon_{a v}(-)$ by another form of the Kozeny-Carman equation:

$\alpha_{a v}=\frac{180\left(1-\varepsilon_{a v}\right)}{\rho_{s}(D[3,2])^{2} \varepsilon_{a v}^{3}}$

The applicability of Kozeny-Carman equation for practical filtration studies is very limited, not least because the PSD and particle shape distributions of solids vary greatly and because the filtered materials tend to be more or less compressible (Svarovsky, 1981; Sorrentino and Anlauf, 2000a,b; Tien and Ramarao, 2013; Tiller, 2004).

The average porosity of the whole filter cake is calculated as

$\varepsilon_{a v}=\frac{V_{v}}{V_{C}}$

where $V_{v}$ is the volume of voids in the cake and $V_{c}$ is the volume of the cake. The volume of the cake was calculated on the basis of its height and cross-sectional area, whereas the void volume was calculated from $V_{C}-V_{S S}$, where $V_{S S}$ is the volume of suspended solids, which in turn was calculated by dividing the mass of dry solids by the density of solids (Table 1).

\section{Results}

\subsection{Reduction of particle size and its effect on filtration and cake properties}

The factors explaining the observed filtration behavior of the ground slurry samples were investigated with respect to various characteristic particle sizes, and the average specific cake resistances $\alpha_{a v}$, which was determined by using the conventional method utilizing averaged filtration data. The plots are presented in Fig. 2 and as can be seen, the fine end of the PSD $\left(D_{10}\right.$, $D[3,2])$ did not have practically any correlation with the average specific resistance to filtration. However, plotting $\alpha_{a v}$ against the $D_{90}$ particle size shows that some interesting phenomena may have occurred as a result of grinding. It seems that insufficient grinding of the largest particles might have an adverse effect regarding filtration, whereas efficient size reduction of the largest particles might reduce the cake resistance. 

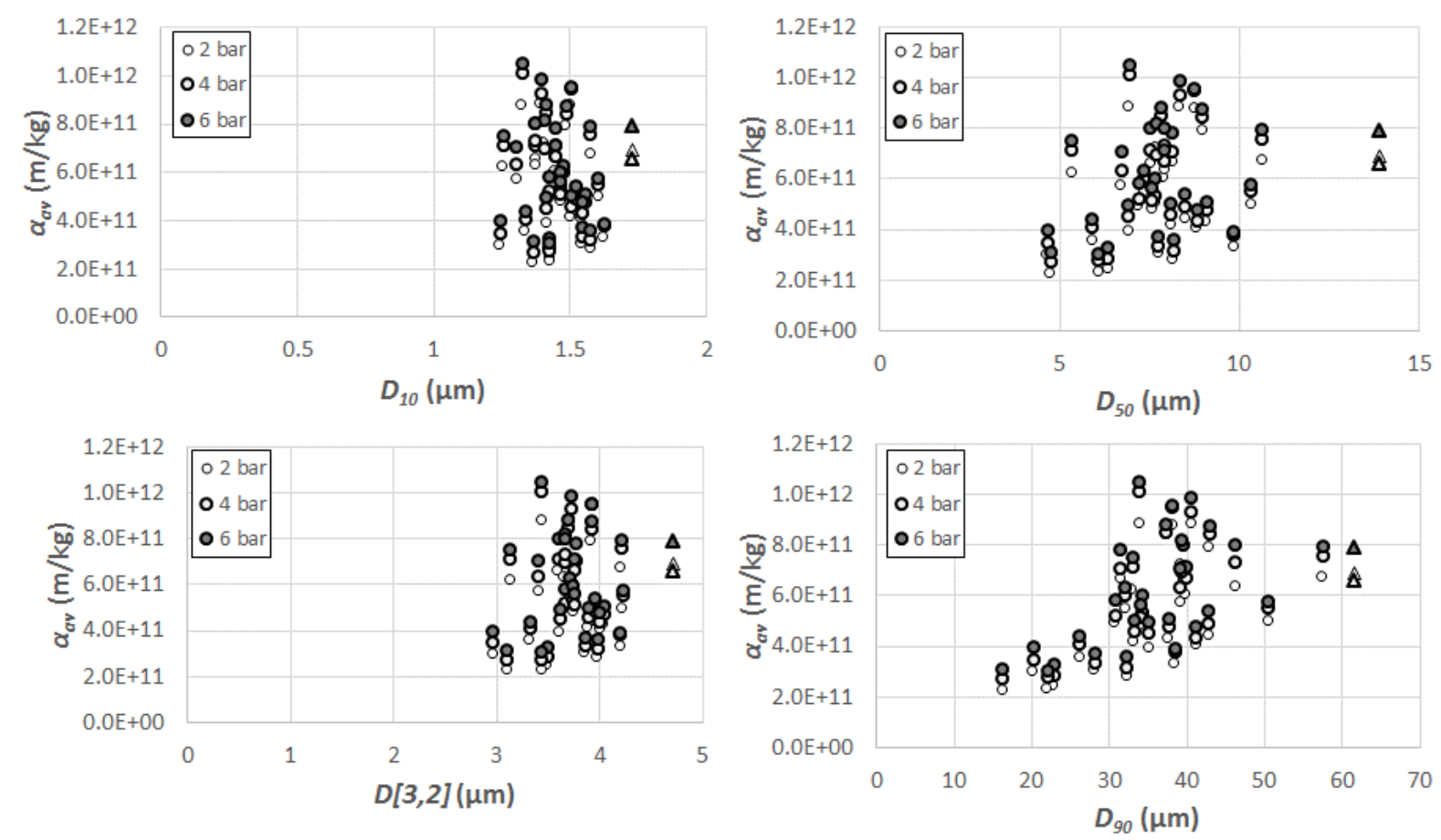

Fig. 2. Average specific cake resistance against different characteristic particle sizes of volumetric undersize distribution. The non-ground samples are marked with triangles.

The same filtration results as above in Fig. 2 are presented as a function of the width of PSD in Fig. 3. Two definitions for the width of PSD are used, the first being the conventional span of size distribution defined as the remainder $\left(D_{90}-D_{10}\right)$ divided by $D_{50}$ (see e.g. Jankovic and Sinclair, 2006; Kinnarinen et al., 2015), and the second one the difference $D[4,3]-D$ [3,2]. It is apparent that $\alpha_{a v}$ does not have a very linear relationship with the width of PSD. The highest values of $\alpha_{a v}$ are obtained, irrespective of the filtration pressure, when the width of PSD is in the middle range. The most important grinding variable resulting in the narrowest PSD is the bead diameter. This result is discussed is closer detail in Section 4.3 below.
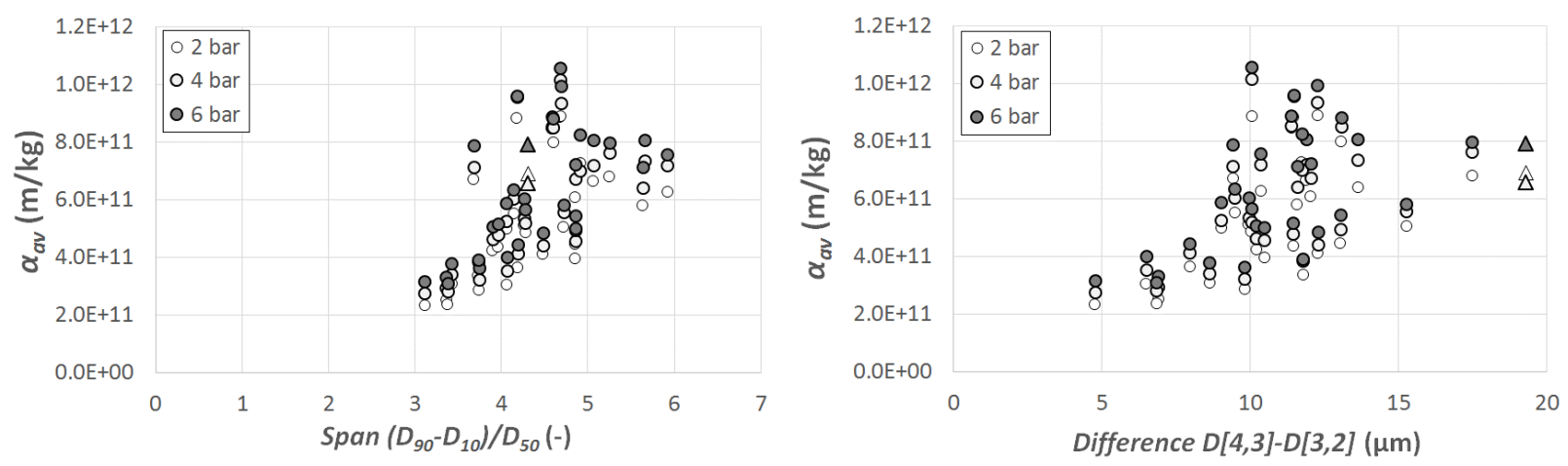
Fig. 3. Average specific cake resistance against the width of the volumetric undersize distribution according to two alternative definitions. The non-ground samples are marked with triangles.

The correlation between $\alpha_{a v}$ and $\varepsilon_{a v}$ of cakes (in the end of each experiment) is illustrated in Fig. 4. It is apparent that low cake porosity is one of the primary reasons for high specific cake resistance, and high porosity enables a lower resistance of the cake. The average porosity of the cake does not, however, depend on the applied pressure difference significantly.

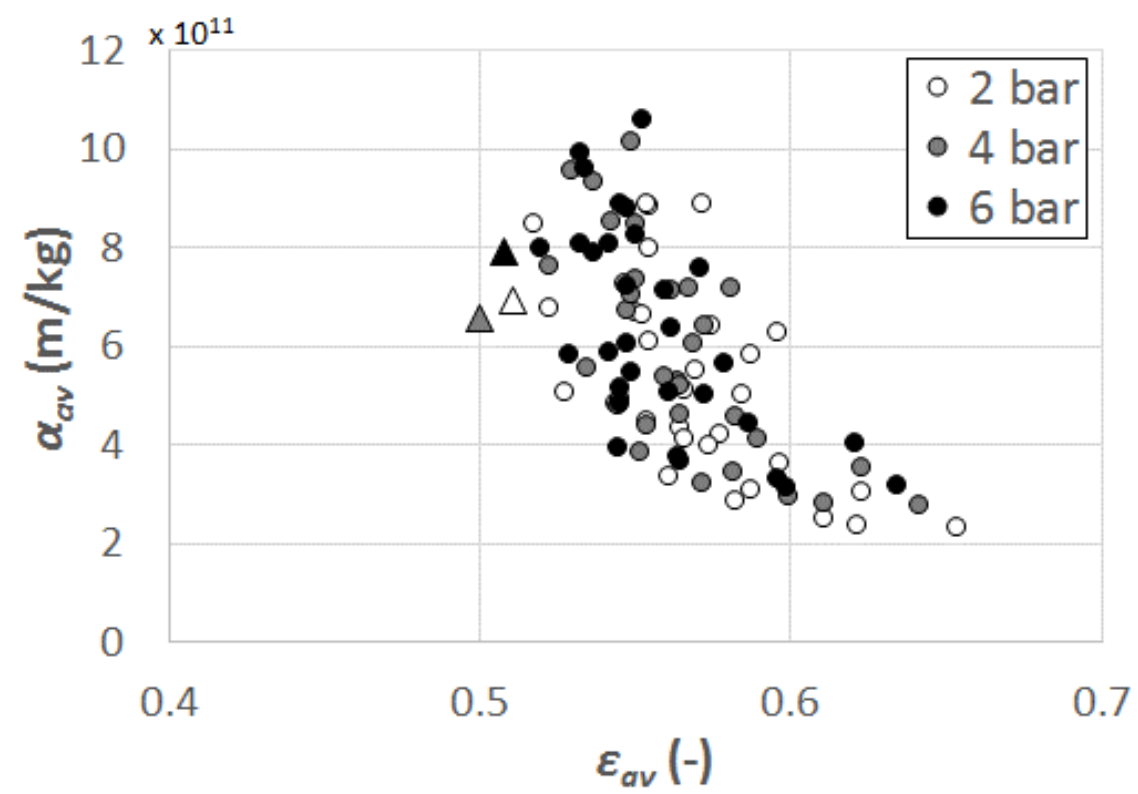

Fig. 4. Correlation between average specific cake resistance and average porosity. The non-ground samples are marked with triangles.

A very clear correlation can be observed when the average porosity $\varepsilon_{a v}$ is presented as a function of the width of PSD (Fig. 5), i.e. the span and the difference $(D[4,3]-D[3,2])$. In fact, the porosity of a compressible cake is lowest at the cake-medium interface and highest at the top of the cake (Tiller and Leu, 1980). The range of the average cake porosities is $0.5-0.66$, and a similar conclusion as above can be drawn here as well: grinding of the solids to obtain a narrow particle size distribution may facilitate filtration, which in this case is closely related to the increased cake porosity. The selection of unsuitable grinding conditions causes formation of a less porous cake in the filtration stage, which in turn hampers the filtration. 

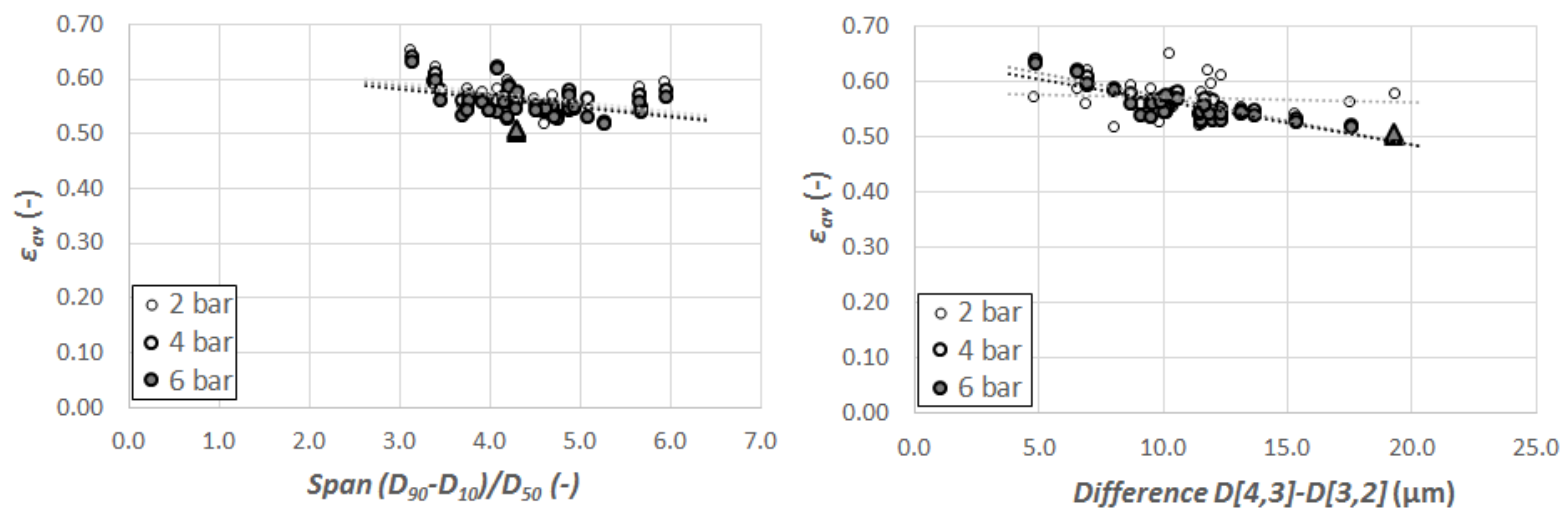

Fig. 5. Average porosity of the filter cake against the width of the volumetric undersize distribution according to two alternative definitions. The non-ground samples are marked with triangles.

The influence of the presence of different particle sizes in the suspension on cake porosity has been conventionally investigated by using binary or ternary mixtures of spherical particles. Ouchlyama and Tanaka (1981) present a replotted figure from an early study of Furnas (1928, 1929), which shows clearly that the porosity of a binary mixture depends on the size ratio and the volumetric fractions of the two size classes. For such mixtures, the minimum bed porosity may be approximately half of the porosity of a bed of monosized particles. Dexter and Tanner (1972) conclude that the packing density of spherical particles increases relatively linearly with the standard deviation of a log-normal PSD within the studied range, which inversely means that the bed porosity decreases as the width of the PSD increases. The same conclusion can be drawn from the results presented by $\mathrm{Yu}$ and Standish (1991).

\section{2. $\quad$ Deeper investigation of particle size distributions}

It was apparent on the basis of the average specific cake resistances and average porosities of the cakes that the improved filtration characteristics were mainly due to the width of PSD. Therefore, the widest and narrowest PSD were further evaluated together with the PSD of the original nonground sample (Table 3 and Fig. 6). As Table 3 shows, the filtration pressure had only a slight effect on the cake porosity. As shown in Fig. 6, the fine end of PSD is not clearly influenced by variation of the grinding conditions. On the contrary, the large end of PSD is clearly dependent on the grinding conditions, in this case on the bead diameter only. Investigation of the filtration characteristics of these experiments ( 6 bar) revealed that increasing the bead diameter from 1 to 3 $\mathrm{mm}$ to obtain a narrower PSD reduced $\alpha_{a v}$ by almost $60 \%$ and increased the corresponding $\varepsilon_{a v}$ by about $11 \%$. However, this result should not be generalized for all types of solids. For instance, Jankovic and Sinclair (2006) did not find the size of the grinding media to be among the most important operational variables, when the aim is to obtain a narrow PSD.

Table 3. The main data of tests 0,4 and 9. Test 0 represents the non-ground sample and the grinding conditions for Tests 4 and 9 are shown in Table 2 above. 


\begin{tabular}{lllll}
\hline $\begin{array}{l}\text { Test } \\
(\#)\end{array}$ & $\begin{array}{l}\text { Filtration pressure } \Delta p \\
(\text { bar })\end{array}$ & $\begin{array}{l}\text { Span }\left(D_{90}-D_{10}\right) / D_{50} \\
(-)\end{array}$ & $\begin{array}{l}\varepsilon_{a v} \\
(-)\end{array}$ & $\begin{array}{l}\alpha_{a v} \\
(\mathrm{~m} / \mathrm{kg})\end{array}$ \\
\hline 0 & 2 & 4.3 & 0.511 & $6.9 \cdot 10^{11}$ \\
0 & 4 & 4.3 & 0.500 & $6.6 \cdot 10^{11}$ \\
0 & 6 & 4.3 & 0.508 & $7.9 \cdot 10^{11}$ \\
4 & 2 & 3.1 & 0.654 & $2.3 \cdot 10^{11}$ \\
4 & 4 & 3.1 & 0.642 & $2.8 \cdot 10^{11}$ \\
4 & 6 & 3.1 & 0.635 & $3.2 \cdot 10^{11}$ \\
9 & 2 & 5.9 & 0.596 & $6.3 \cdot 10^{11}$ \\
9 & 4 & 5.9 & 0.581 & $7.2 \cdot 10^{11}$ \\
9 & 6 & 5.9 & 0.571 & $7.6 \cdot 10^{11}$ \\
\hline
\end{tabular}

The result presented above, referring to Figs. 5 and 6, is in good agreement with theories presented on cake formation in the literature. It is typically assumed (see e.g. Hwang et al., 1997; Koekemoer and Luckos, 2015) that a wide PSD will usually cause more complete filling of voids between larger particles in the cake by smaller particles. A narrow PSD, on the other hand, indicates a more uniform particle size, which contributes to the formation of an open cake structure with lower resistance to fluid flow.

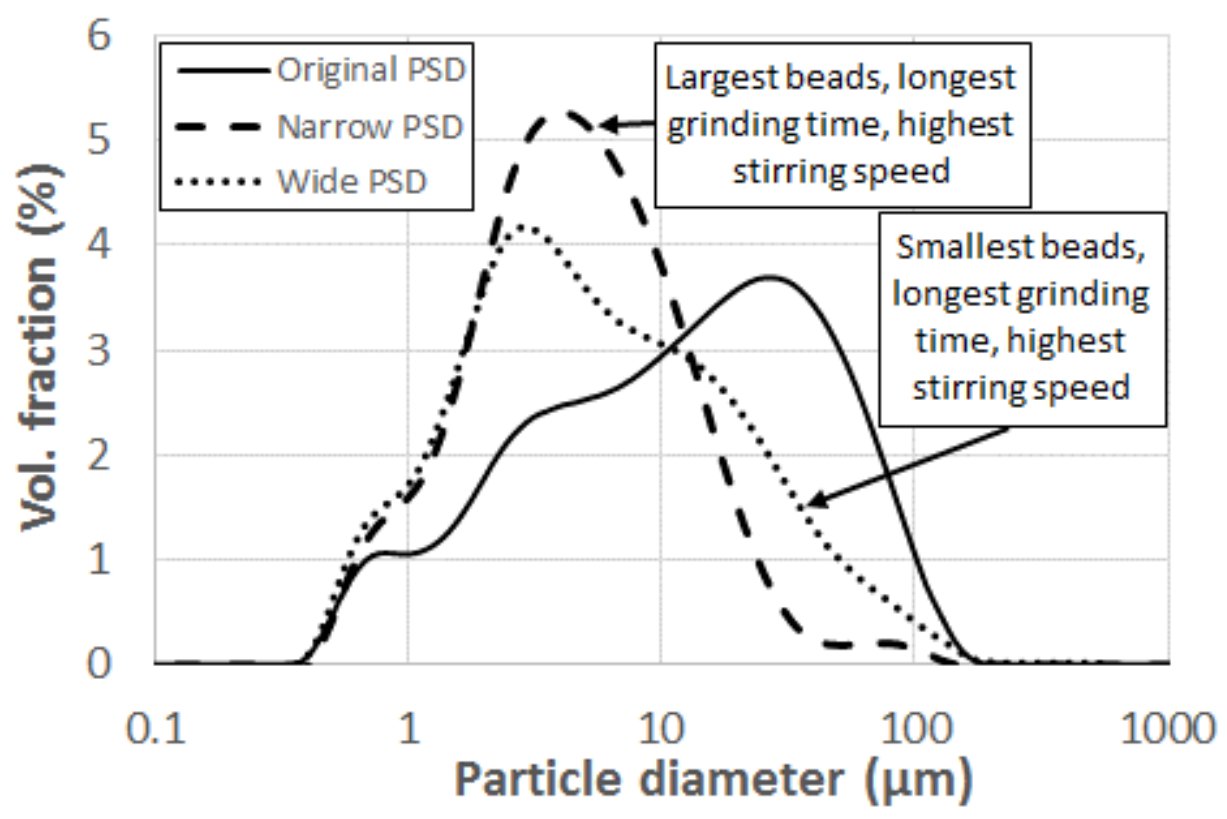

Fig. 6. Differential volumetric PSD of the original sample, as well as the narrowest (Test 4) and the widest (Test 9) PSDs.

\subsection{Relative effect of each grinding variable on the average specific cake resistance}

A straightforward linear regression analysis was performed in order to evaluate the impact of the experimental variables (without interaction terms) on the specific resistance to filtration. The 
equation is of the form $y=\beta_{0}+\beta_{1} X_{1}+\beta_{2} X_{2}+\beta_{3} X_{3}+\beta_{4} X_{4}$, where $y$ is the predicted specific cake resistance, $\beta_{1^{-}} \beta_{4}$ are the coefficients of variables $X_{1}-X_{4}$, and $\beta_{0}$ represents the intercept (Table 4). Prior to the regression analysis, the values of the variables were normalized by using the range -1 to 1 . The strength and direction of the effect of each variable is clear to see by comparing the values of the coefficients which are summarized in Table 4, together with the most important statistical values.

Table 4. Results of regression analysis for the prediction of $\alpha_{a v}$ with normalized values of variables $X_{1}-X_{4}\left(R^{2}=0.89\right)$.

\begin{tabular}{llll}
\hline Variable & $\begin{array}{l}\text { Coefficient } \beta_{i} \\
\left(\cdot 10^{11}\right)\end{array}$ & $\begin{array}{l}\text { Mean error } \\
\left(\cdot 10^{9}\right)\end{array}$ & P-value \\
\hline Bead diameter $\left(X_{1}\right)$ & -1.96 & 9.45 & $1.38 \cdot 10^{-35}$ \\
Grinding time $\left(X_{2}\right)$ & -0.34 & 9.45 & $4.81 \cdot 10^{-4}$ \\
Stirring speed $\left(X_{3}\right)$ & -1.59 & 9.45 & $2.92 \cdot 10^{-29}$ \\
Filtration pressure $\left(X_{4}\right)$ & 0.48 & 8.82 & $4.68 \cdot 10^{-7}$ \\
Intercept & 5.79 & 7.2 & $3.83 \cdot 10^{-84}$ \\
\hline
\end{tabular}

The squared Pearson correlation coefficient $R^{2}$ (see Rodgers and Nicewander (1988) for definition) for the regression model is 0.89 , which can be regarded as satisfactory for a linear model. Comparison between the coefficients presented in Table 4 shows that the bead diameter has the strongest influence on the filterability of the ground tailings slurry within the investigated range of the variables and that the effect of stirring speed is strong as well. The results of regression analysis suggest that increasing both the bead diameter and the stirring speed make the filtration of this tailings slurry easier. The use of a long grinding time can also facilitate filtration, although the effect is not very distinct in this case. Due to the slight compressibility of the filter cakes, the average specific cake resistance can be seen to increase with the filtration pressure. The small Pvalues ( $<0.001$ in all cases) indicate good statistical significance of the variables included in the regression model, in other words, good ability of the variables to explain the observed correlation. Correlation between the measured and predicted values of the average specific cake resistance is illustrated in Fig. 7. 


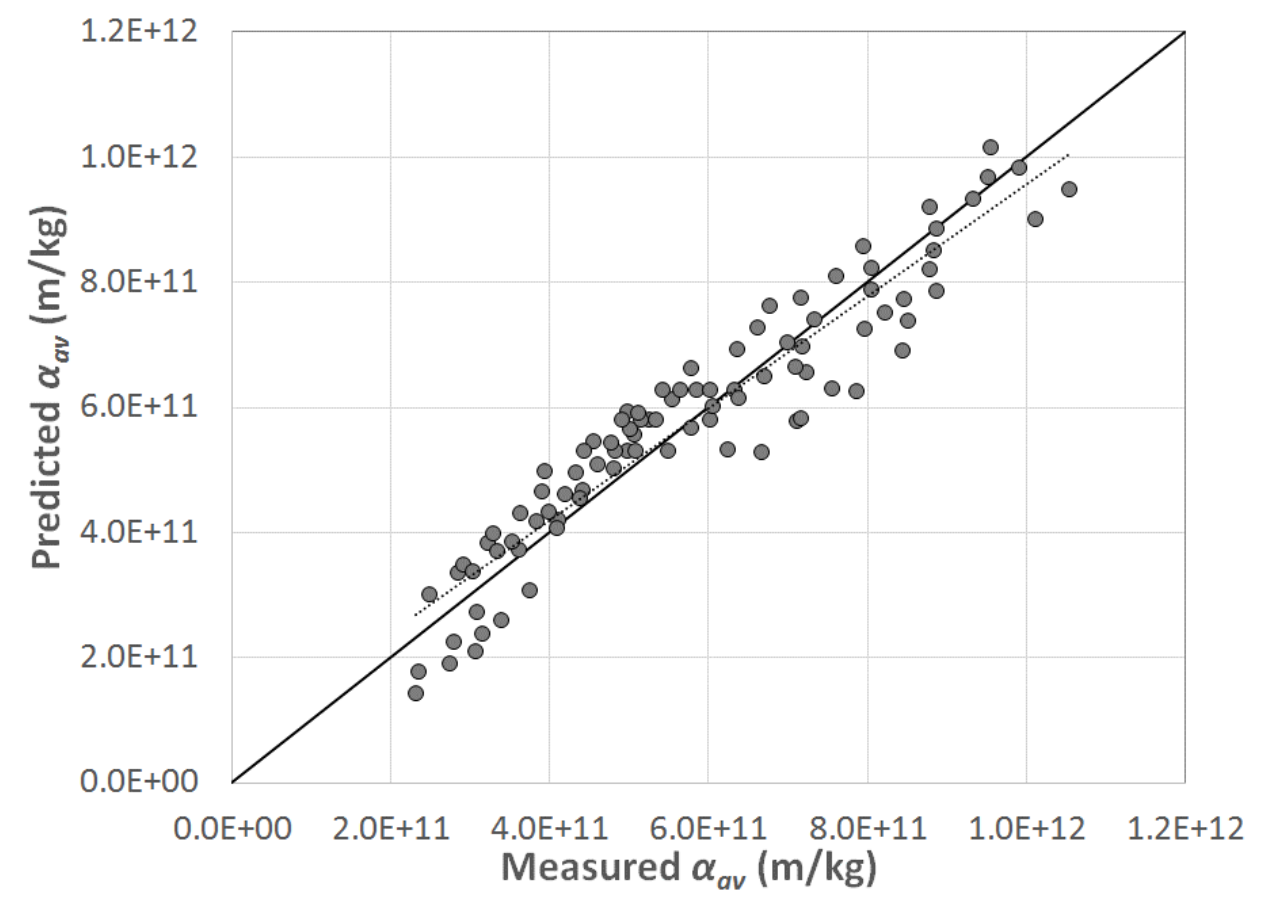

Fig. 7. Measured and predicted average specific cake resistances $\alpha_{a v}$ for the ground samples, the fitted linear function $y=a x+b$ (dashed line), and the diagonal line describing ideal correlation.

\subsection{Changes in particle morphology}

Two ground samples and the original sample, the particle size distributions of which were presented above in Fig. 6, were selected for morphological investigation with Malvern Morphologi G3. The device is capable of performing automated image analysis for a large number of particles, and the number of measured particles was $45000-71000$ per sample. The objective was to evaluate whether the changes in the particle shape could explain the observed changes in the filtration properties. Fig. 8 illustrates the particle shapes of the non-ground material and the ground solids having the narrowest and the widest PSD.

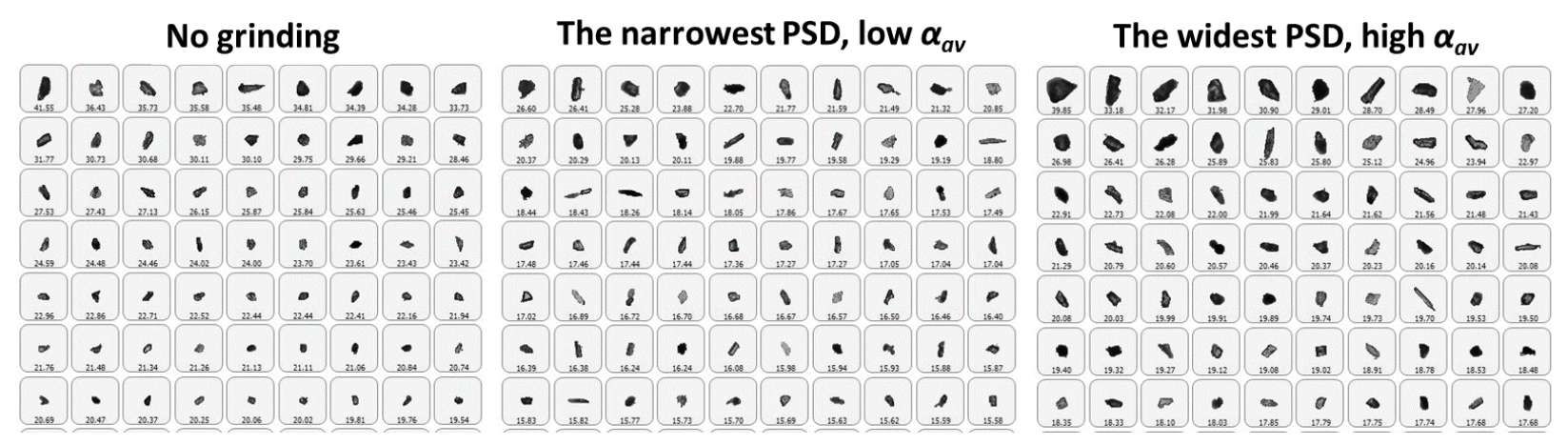

Fig. 8.

Illustration of particle shapes of the selected samples. Note that the scale of the images varies, i.e. the particle sizes are not comparable between the samples. 
Fig. 9 shows volumetric distributions with respect to two shape factors of the particles, namely elongation and HS (High Sensitivity) circularity. Elongation is classified as a one-dimensional measure of particle shape, while the HS circularity, also called Cox circularity, is a twodimensional shape factor (Bagheri et al., 2015). Elongation, which is defined as 1 - (aspect ratio), describes how elongated a particle is, so for perfectly spherical or cubical particles elongation equals zero. The HS circularity is defined as $4 \pi A / P^{2}, A$ and $P$ representing the particle area and perimeter, respectively. Unlike elongation, HS circularity depends on the surface roughness of the particle and is 1 when the particle is a smooth sphere.

As can be seen in the illustrations shown in Figs. 8 and 9, grinding did not cause dramatic changes in the particle morphology. The clearest differences between the shape distributions, especially in Fig. 9b, are visible but do not perhaps enable making definitive conclusions regarding filtration properties.

In the literature, spherical particles have been reported to form a cake with low porosity (Bourcier et al., 2016), compared to most but not all non-spherical shapes of particles, and the specific surface area and the specific cake resistance become higher as well, compared to e.g. cylindrical and rectangular particles (Wakeman, 2007). The porosity of the filter cake is influenced e.g. by the shape and surface roughness of the particles.
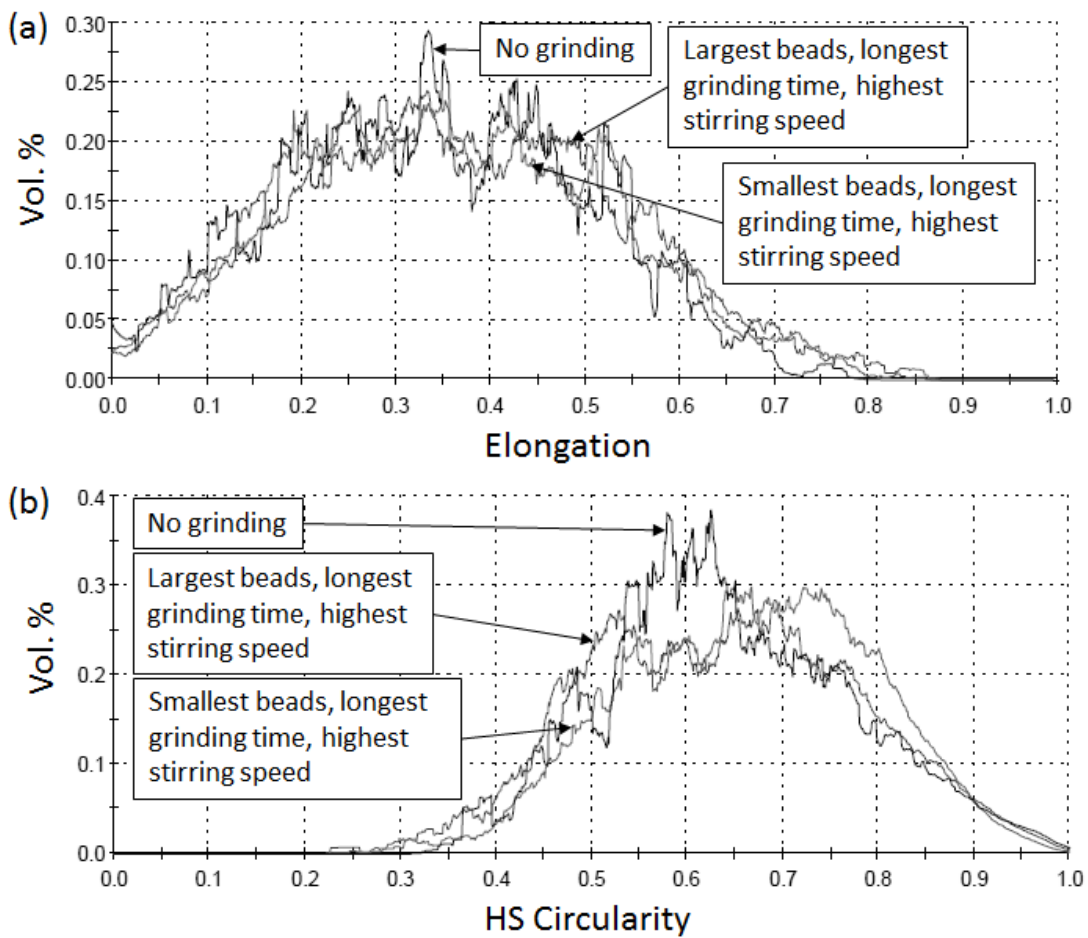

Fig. 9. $\quad$ Elongation (a) and HS circularity (b) distributions of the original tailings particles and the particles having the narrowest and widest size distributions after grinding (Tests 4 and 9, see Fig. 6).

\section{Conclusions}


The effect of stirred media grinding conditions on the pressure filtration characteristics of $\mathrm{Ni}-\mathrm{Cu}$ mine tailings were investigated in this experimental study. The results showed that bead size had the strongest impact on filterability with regard to the average specific cake resistance. Extending the duration of grinding and increasing the stirring rate had also a positive effect on the filterability, although the particle size was reduced in all grinding experiments. The pressure filtration results were relatively poorly explained by any single characteristic particle size. However, the width of particle size distribution correlated clearly with the average specific cake resistance, and even better with the porosity of the cake. The characterization of particle morphology of the original and ground samples did not expose critical changes in the elongation and circularity of the particles. The regression analysis showed that the effect of grinding conditions on filtration performance was significantly stronger than the effect of filtration pressure within the selected range of experimental variables. Evaluation of the obtained particle size distributions implied that size reduction of the largest particles was mainly responsible for the ease of pressure filtration. The high importance of the bead diameter comes from the fact that large beads are more effective in the grinding of coarse particles, the size of which should be reduced to obtain a highly porous structure of the cake. The utilization potential of the results of this study in existing industrial processes is highly dependent on the material properties and the possibilities to modify the plant flowsheet. Therefore, the results should not be generalized to different materials and processes.

\section{References}

Allen, T., 2003. Powder Sampling and Particle Size Determination, Elsevier B.V., Amsterdam

Bagheri, G.H., Bonadonna, C., Manzella, I., Vonlanthen, P., 2015. On the characterization of size and shape of irregular particles. Powder Technol. 270, 141-153.

Basim, G.B., Khalili, M., 2015. Particle size analysis on wide size distribution powders; effect of sampling and characterization technique. Adv. Powder Technol. 26, 200-207.

Bourcier, D., Feraud, J.P., Colson, D., Mandrick, K., Ode, D., Brackx, E., Puel, F., 2016. Influence of particle size and shape properties on cake resistance and compressibility during pressure filtration. Chem. Eng. Sci. 144, 176-187.

Concha, F., 2014. Solid-Liquid Separation in the Mining Industry. Springer International Publishing, Switzerland.

Darcy, H. P. G., 1856. Les Fontaines Publiques de la Ville de Dijon. Victor Dalamont, Paris. Dell, C. C. and Sinha, J., 1964. The mechanism of removal of water from flocculated clay sediments. Trans. Br. Ceram. Soc. 63, 603-614.

Dexter, A.R., Tanner, D.W., 1972. Packing densities of mixtures of spheres with log-normal size distributions. Nature Phys. Sci. 238, July 10, 1972.

Furnas, C.C., 1928. The relations between specific volume, voids, and size composition in systems of broken solids of mixed sizes. Bur. Mines Rep. Invest. 2894 (7), 1-10. 
Furnas, C.C., 1929. Fluid flow through beds of broken solids. Bull. Bur. Mines 307, 74.

Hwang, K.-J., Wu, Y.-S., Lu, W.-M., 1997. Effect of the size distribution of spheroidal particles on the surface structure of a filter cake. Powder Technol. 91, 105-113.

Jankovic, A., Sinclair, S., 2006. The shape of product size distributions in stirred mills. Miner. Eng. $19,1528-1536$.

Kinnarinen, T., Tuunila, R., Huhtanen, M., Häkkinen, A., Kejik, P., Sverak, T., 2015. Wet grinding of $\mathrm{CaCO}_{3}$ with a stirred media mill: Influence of obtained particle size distributions on pressure filtration properties. Powder Technol. 273, 54-61.

Koekemoer, A., Luckos, A., 2015. Effect of material type and particle size distribution on pressure drop in packed beds of large particles: Extending the Ergun equation. Fuel 158, 232-238.

Lin, C.L., Miller, J.D., 2000a. Network analysis of filter cake pore structure by high resolution Xray microtomography. Chem. Eng. J. 77, 79-86.

Lin, C.L., Miller, J.D., 2000b. Pore structure and network analysis of filter cake. Chem. Eng. J. 80, 221-231.

Lin, C.L., Miller, J.D., 2004. Pore structure analysis of particle beds for fluid transport simulation during filtration. Int. J. Miner. Process. 73, 281-294.

Osterroth, S., Preston, C., Markicevic, B., Iliev, O., Hurwitz, M., 2016. The permeability prediction of beds of poly-disperse spheres with applicability to the cake filtration. Sep. Purif. Technol. 165, $114-122$.

Ouchiyama, N., Tanaka, T., 1981. Porosity of a mass of solid particles having a range of sizes, Ind. Eng. Chem. Fundam. 20 (1), 66-71.

Ripperger, S., Gösele, W., Alt, C. 2012. Filtration, 1. Fundamentals, In: Ullmann's Encyclopedia of Industrial Chemistry, Wiley-VCH Verlag GmbH \& Co. KgaA, Weinheim.

Rodgers, J.L., Nicewander, W.A. 1988. Thirteen ways to look at the correlation coefficient, Am. Stat. 42, 59-66.

Sorrentino, J.A., Anlauf, H., 2000a. Influence of particle size distribution on cake permeability. European Federation of Chemical Engineering, Brighton, UK.

Sorrentino, J.A., Anlauf, H., 2000b. The use of particle size distribution for estimating cake permeability. Proceedings of the 2000 Annual Technical Conference of the American Filtration \& Separation Society, Adv. Filtr. Sep. Technol., vol. 14.

Svarovsky, L., 1981. Solid-liquid Separation, second ed., Butterworth \& Co, Witham, Essex. 
Tarleton, S., Wakeman, R., 2007. Solid/liquid separation: equipment selection and process design. Butterworth-Heinemann, Oxford.

Tien, C., 2012. Principles of filtration. Elsevier, Oxford.

Tien, C., Ramarao, V., 2013. Can filter cake porosity be estimated based on the Kozeny-Carman equation? Powder Technol. 237, 233-240.

Tiller, F.M. 2004. What the filter man should know about theory. Filtration, Special Issue, April 2004, 55-67.

Tiller, F.M., Leu, W.-F., 1980. Basic data fitting in filtration. J. Chin. Inst. Chem. Eng. 11, 61-70.

Wakeman, R., The influence of particle properties on filtration. Sep. Purif. Technol. 58, 234-241.

Yu, A.B., Standish, N., 1991. Estimation of the porosity of particle mixtures by a linear mixture packing model. Ind. Eng. Chem. Res. 30(6), 1372-1385. 